\title{
Add-on Therapy With the a-Blockers Tamsulosin and Naftopidil Improves Voiding Function by Enhancing Neuronal Activity in Prostatic Hyperplasia Rats
}

\author{
Il-Gyu Ko ${ }^{1}$, Lakkyong Hwang ${ }^{1}$, Jun-Jang Jin ${ }^{1}$, Sang-Hoon Kim ${ }^{1}$, Jin Hee Han², Jung Won Jeon ${ }^{3}$, Sung Tae Cho ${ }^{4}$ \\ ${ }^{1}$ Department of Physiology, College of Medicine, Kyung Hee University, Seoul, Korea \\ ${ }^{2}$ Department of Anesthesiology and Pain Medicine, Kyung Hee Medical Center, Kyung Hee University College of Medicine, Seoul, Korea \\ ${ }^{3}$ Department of Internal Medicine, Kyung Hee University Hospital at Gandong, Kyung Hee University College of Medicine, Seoul, Korea \\ ${ }^{4}$ Department of Urology, Hallym University Kangnam Sacred Heart Hospital, Hallym University College of Medicine, Seoul, Korea
}

Purpose: Benign prostatic hyperplasia (BPH) impacts quality of life in men by causing lower urinary tract symptoms. a1Adrenoceptor ( $a 1-\mathrm{AR})$ blockers improve lower urinary tract symptoms. We investigated the efficacy of add-on therapy with a1-AR blockers on BPH rats.

Methods: Rats in the drug-treated groups were orally administered each drug once a day for 30 days after orchiectomy. To induce $\mathrm{BPH}$, rats were castrated and testosterone $(20 \mathrm{mg} / \mathrm{kg})$ was injected subcutaneously once per day for 30 days. Cystometry was conducted to measure voiding contraction pressure and the interval contraction time, immunohistochemistry was performed to measure c-Fos and nerve growth factor (NGF) expression in the neuronal voiding centers, and nicotinamide adenine dinucleotide phosphate-diaphorase histochemistry was used to measure nitric oxide synthase (NOS) expression.

Results: Orchiectomy and testosterone injection decreased voiding contraction pressure and the interval contraction time, suggesting BPH symptoms. Voiding contraction pressure and the interval contraction time were greater in the group that received the combination treatment (tamsulosin with naftopidil) than in the tamsulosin monotherapy or naftopidil monotherapy groups. c-Fos, NGF, and NOS expression in the neuronal voiding centers was enhanced by BPH induction. c-Fos, NGF, and NOS expression was suppressed by the combination treatment (tamsulosin with naftopidil) to a greater extent than was the case for tamsulosin monotherapy or naftopidil monotherapy.

Conclusions: Combination therapy of tamsulosin and naftopidil showed greater efficacy for the treatment of BPH than tamsulosin monotherapy or naftopidil monotherapy; therefore, combination therapy can be considered as a novel therapeutic method for BPH.

Keywords: Prostatic hyperplasia; Combined therapy; Neuronal voiding center; c-fos; Nerve growth factor; Nitric oxide synthase

- Grant/Fund Support: This research was supported by a grant from the National Research Foundation of Korea (NRF-2014R1A2A1A11052321). This research was supported by Hallym University Research Fund 2016 (HURF-2016-15).

- Research Ethics: All animal procedures complied with the Institutional Care and Use Committee (KHUASP[SE]-14-047) of Kyung Hee University and were performed in accordance with the guiding principles for the care and use of animals approved by the Council of the National Institutes of Health Guide for the Care and Use of Laboratory Animals.

- Conflict of Interest: No potential conflict of interest relevant to this article was reported.

\section{- HIGHLIGHTS}

- As combination therapy showed greater efficacy for the treatment of BPH than monotherapy, combination therapy can be considered a new therapeutic method for $\mathrm{BPH}$.

Corresponding author: Sung Tae Cho (iD https://orcid.org/0000-0002-4691-6159 Department of Urology, Hallym University Kangnam Sacred Heart Hospital, Hallym University College of Medicine, 1 Singil-ro, Yeongdeungpo-gu, Seoul 07441, Korea

E-mail: cst326@paran.com / Tel: +82-2-829-5326 / Fax: +82-2-846-5198

Submitted: March 19, 2018 / Accepted after revision: March 26, 2018
This is an Open Access article distributed under the terms of the Creative Commons Attribution Non-Commercial License (http://creativecommons.org/licenses/by-nc/4.0/) which permits unrestricted non-commercial use, distribution, and reproduction in any medium, provided the original work is properly cited. 


\section{INTRODUCTION}

Benign prostatic hyperplasia $(\mathrm{BPH})$, which is common in older men, is an enlargement of the prostate gland that may lead to bladder outlet obstruction, lower urinary tract symptoms, and reduced quality of life [1]. Normal bladder filling and storage processes require accommodation for increasing urine volume under low intravesical pressure with appropriate sensation. The sphincter remains closed as intra-abdominal pressure increases and involuntary bladder contractions do not occur [2]. However, patients with $\mathrm{BPH}$ show voiding and storage symptoms due to bladder outlet obstruction following enlargement of the prostate [3,4].

Bladder outlet obstruction caused by BPH is associated with marked alterations in bladder structure and function, including detrusor overactivity, with symptoms of urinary frequency, urgency, and urge incontinence [5]. More than half of patients with $\mathrm{BPH} /$ lower urinary tract symptoms have overactive bladder symptoms. The management of BPH includes nonpharmacological, pharmacological, and surgical interventions, and the choice of therapy depends on the pattern and severity of symptoms. Although surgery is the definitive treatment for $\mathrm{BPH}$, the risks, complications, and costs of surgical intervention suggest the need for effective and safe noninvasive treatments, such as drug therapy. The pharmacological management of lower urinary tract symptoms by BPH includes several categories of drugs with different modes of action.

a1-Adrenoceptor ( $\alpha 1$-AR) blockers have been widely used to treat $\mathrm{BPH}$, as they decrease muscle tone and inhibit smooth muscle contraction by blocking adrenergic receptors, which are abundant in the bladder neck, prostatic capsule, and prostatic tissue [6]. Thus, a1-AR blockers are first-line treatments and have been shown to be effective and safe $[7,8]$. Recently, to increase their therapeutic effects, $\alpha 1$-AR blockers have been used to control BPH in the form of combination therapy, as well as monotherapy. Combination therapy is particularly promising for improving BPH-induced lower urinary tract symptoms.

Therefore, we compared the effect of combination therapy with a1-AR blockers (tamsulosin with naftopidil) to the effect of tamsulosin monotherapy or naftopidil monotherapy using $\mathrm{BPH}$ rats. In this study, cystometry was performed, as well as immunohistochemistry for c-Fos and nerve growth factor (NGF) and histochemistry for nicotinamide adenine dinucleotide phosphate-diaphorase (NADPH-d) in the neuronal voiding centers (medial preoptic area [MPA], ventrolateral periaq- ueductal gray [vlPAG], pontine micturition center [PMC], and spinal cord L4-L5).

\section{MATERIALS AND METHODS}

\section{Animals and Treatments}

Adult male Sprague-Dawley rats, weighing $210 \pm 5$ g (9 weeks old), were used for the experiments. All animal procedures complied with the regulations of the Institutional Care and Use Committee (KHUASP[SE]-14-047) of Kyung Hee University and were performed in accordance with the guiding principles for the care and use of animals approved by the Council of the National Institutes of Health Guide for the Care and Use of Laboratory Animals. The rats were housed under controlled temperature $\left(23^{\circ} \mathrm{C} \pm 2^{\circ} \mathrm{C}\right)$ and lighting $\left(8_{\mathrm{AM}}\right.$ to $\left.9_{\mathrm{PM}}\right)$ conditions with food and water available ad libitum. The animals were randomly divided into the following 5 groups $(\mathrm{n}=10$ in each group): the sham operation group, the $\mathrm{BPH}$-induced group, the $\mathrm{BPH}$-induced and tamsulosin-treated group, the $\mathrm{BPH}$-induced and naftopidil-treated group, and the BPH-induced and combination-treated group.

The recommended daily allowance of each drug was administered as follows: $0.2 \mathrm{mg} / \mathrm{kg}$ of tamsulosin (Harnal, Astellas Pharma, Inc., Tokyo, Japan), 75 mg/kg of naftopidil (Flivas, Asahi Kasei Pharma, Tokyo, Japan), and $0.2 \mathrm{mg} / \mathrm{kg}$ of tamsulo$\sin +75 \mathrm{mg} / \mathrm{kg}$ of naftopidil in the combination therapy group. Rats in the drug-treated groups orally received each drug once a day for 30 consecutive days after orchiectomy. The animals in the control and $\mathrm{BPH}$-induced groups received the same amount of distilled water.

\section{Induction of $\mathrm{BPH}$}

The $\mathrm{BPH}$ animal model was made following a previously described method [9]. The rats were anesthetized with Zoletil 50 (50 mg/kg; Virbac Laboratories, Carros, France). The testes and epididymis were exposed through a midline ventral scrotal incision, and the extratesticular rete testis together with the pampiniform plexus vessels were ligated. Then, the testes were removed, and the ligated efferent ductules and epididymis were returned to the scrotum. In the sham-operation rats, the testes were exposed, manipulated, and then reinserted into the scrotum. After surgery, the scrotal incision was closed by suturing. After orchiectomy, BPH was induced by a subcutaneous injection of testosterone $(20 \mathrm{mg} / \mathrm{kg}$; Wako Pure Chemical Industries, Ltd., Osaka, Japan) for 30 days. 


\section{Cystometry}

Voiding contraction pressure and the interval contraction time were determined following a previously described method $[10,11]$. Cystometry was performed 32 days after orchiectomy. The rats were anesthetized with Zoletil $50(10 \mathrm{mg} / \mathrm{kg}$, interperitoneally; Virbac Laboratories). A sterile polyethylene catheter (PE50) with a cuff was implanted in the bladder through an abdominal midline incision into the dome and held in place by a purse-string suture. The catheter was connected to a pressure transducer (Harvard Apparatus, Holliston, MA, USA) and syringe pump (Harvard Apparatus) via a 3-way stopcock to record the intravesical pressure and to infuse saline into the bladder. After the bladder was emptied, cystometry was performed by infusing $0.5 \mathrm{~mL}$ of saline. Voiding contraction pressure and interval contraction time in the bladder were monitored using Labscribe (iWork System Inc., Dover, NH, USA).

\section{Tissue Preparation}

The animals were sacrificed immediately after evaluating their voiding function by cystometry, following a previously described method $[10,12]$. The rats were anesthetized using Zoletil 50 (10 mg/kg, interperitoneally; Virbac Laboratories), transcardially perfused with $50 \mathrm{mM}$ phosphate-buffered saline (PBS), and fixed with freshly prepared $4 \%$ paraformaldehyde in $100 \mathrm{mM}$ phosphate buffer (pH, 7.4). The brain and spinal cord were dissected and stored overnight in the same fixative, and then transferred into 30\% sucrose for cryoprotection. For immunohistochemistry, the slices were coronally sectioned at a thickness of $40 \mu \mathrm{m}$ using a cryostat (Leica, Wetzlar, Germany). The PMC was selected from the region spanning from Bregma -9.68 to $-9.80 \mathrm{~mm}$, the vlPAG was selected from the region spanning from Bregma -7.64 to $8.00 \mathrm{~mm}$, the MPA was selected from the region spanning from Bregma -0.26 to $0.80 \mathrm{~mm}$, and the spinal cord was selected from the L4 -5 regions. On average, 10 sections in each region were collected from each rat.

\section{Immunohistochemistry for c-Fos and NGF}

An immunohistochemical analysis was conducted to evaluate c-Fos and NGF expression in the MPA, vlPAG, PMC, and spinal cord L4-5 regions, following a previously described method $[10,12]$. Free-floating tissue sections were incubated overnight with rabbit anti-c-Fos and mouse anti-NGF antibodies (1:1,000; Santa Cruz Biotechnology, Santa Cruz, CA, USA) and the sections were incubated for 1 hour with biotinylated anti-rabbit cFos and anti-mouse NGF secondary antibodies (1:200; Vector
Laboratories, Burlingame, CA, USA). Next, the sections were incubated with avidin-biotin-peroxidase complex (Vector Laboratories) for 1 hour at room temperature. For staining, the sections were incubated in $0.03 \% \mathrm{DAB}$ and $0.03 \%$ hydrogen peroxide for 5 minutes. The sections were mounted onto gelatincoated slides. The slides were air-dried overnight at room temperature, and coverslips were mounted using Permount (Fisher Scientific, Waltham, MA, USA).

\section{Histochemistry for NADPH-d}

To measure nitric oxide synthase (NOS) activity in the neuronal voiding centers, histochemistry for NADPH-d was performed following a previously described method [10]. Briefly, free-floating sections were incubated at $37^{\circ} \mathrm{C}$ for 60 minutes in $100 \mathrm{mM}$ PBS (pH, 7.4) containing 0.3\% Triton X-100, 0.1-mg/ $\mathrm{mL}$ nitroblue tetrazolium, and $0.1-\mathrm{mg} / \mathrm{mL} \beta-\mathrm{NADPH}$. The sections were washed 3 times with PBS and mounted onto gelatincoated slides. The slides were air-dried overnight at room temperature, and coverslips were mounted using Permount (Fisher Scientific).

\section{Data Analysis}

The numbers of c-Fos-positive, NGF-positive, and NADPH-dpositive cells in the MPA, vlPAG, PMC, and spinal cord L4-L5 regions were counted hemilaterally through a light microscope (Olympus, Tokyo, Japan). The area of the MPA, PMC, vlPAG, and spinal cord L4 -5 regions from each slice was measured using an Image-Pro Plus computer-assisted image analysis system (Media Cyberbetics Inc., Silver Spring, MD, USA) attached to a light microscope (Olympus).

Statistical analysis was performed using 1-way analysis of variance followed by the Duncan post hoc test, and the results are expressed as the mean \pm standard error of the mean. Significance was set at $\mathrm{P}<0.05$.

\section{RESULTS}

\section{Voiding Contraction Pressure and Interval Contraction Time} The voiding contraction pressure and interval contraction time from cystometry are presented in Fig. 1. These results show that voiding contraction pressure and the interval contraction time were significantly decreased by the induction of $\mathrm{BPH}(\mathrm{P}<0.05)$. Monotherapy with tamsulosin or naftopidil did not exert a significant effect on voiding contraction pressure or the interval contraction time, whereas combination therapy (tamsulosin 

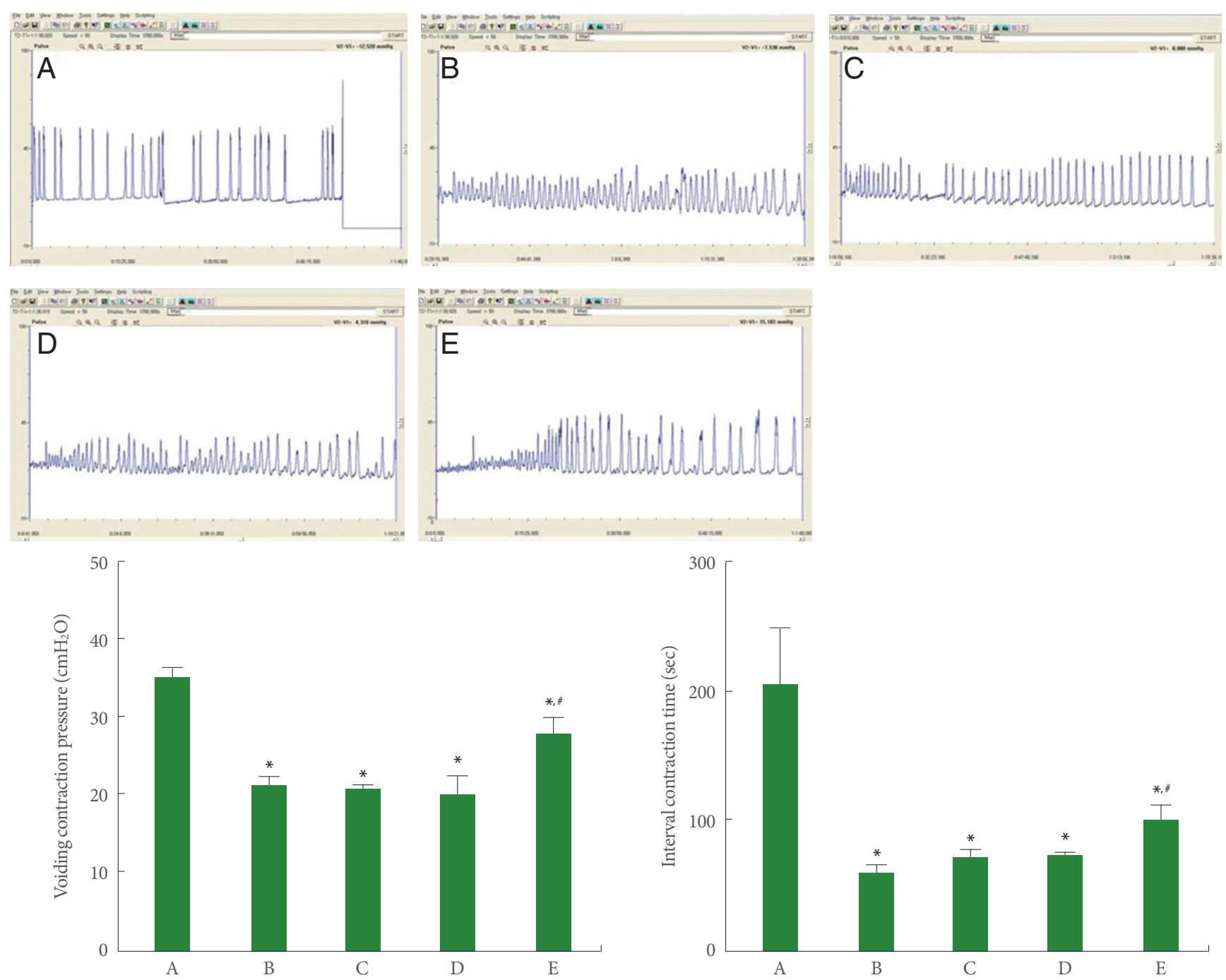

Fig. 1. Voiding contraction pressure and interval contraction time in bladder. Upper panel: The voiding contraction pressure and interval contraction time change in the cystometry. Lower panel: Comparison of voiding contraction pressure (left) and interval contraction time (right) in each group. A, sham-operation group; B, benign prostatic hyperplasia (BPH)-induced group; C, $\mathrm{BPH}$-induced and tamsulosin-treated group; D, BPH-induced and naftopidil-treated group; E, BPH-induced and combination-treated group. ${ }^{*} \mathrm{P}<$ 0.05 compared to the sham-operation group. ${ }^{*} \mathrm{P}<0.05$ compared to the $\mathrm{BPH}$-induced group.

with naftopidil) significantly increased voiding contraction pressure and the interval contraction time in the $\mathrm{BPH}$ rats $(\mathrm{P}<0.05)$.

\section{c-Fos Expression in Neuronal Voiding Centers}

Photomicrographs of c-Fos expression in the neuronal voiding centers are presented in Fig. 2. The present results show that cFos expression in neuronal voiding centers was significantly increased by the induction of BPH $(\mathrm{P}<0.05)$. Monotherapy with tamsulosin or naftopidil showed an inhibitory effect on c-Fos expression depending on the site in the neuronal voiding centers of $\mathrm{BPH}$ rats $(\mathrm{P}<0.05)$. Combination therapy (tamsulosin with naftopidil) led to an even greater reduction in c-Fos expression at all sites of the neuronal voiding centers of $\mathrm{BPH}$ rats $(\mathrm{P}<0.05)$.

\section{NGF Expression in Neuronal Voiding Centers}

Photomicrographs of NGF expression in the neuronal voiding centers are presented in Fig. 3. The present results show that NGF expression in neuronal voiding centers was significantly 

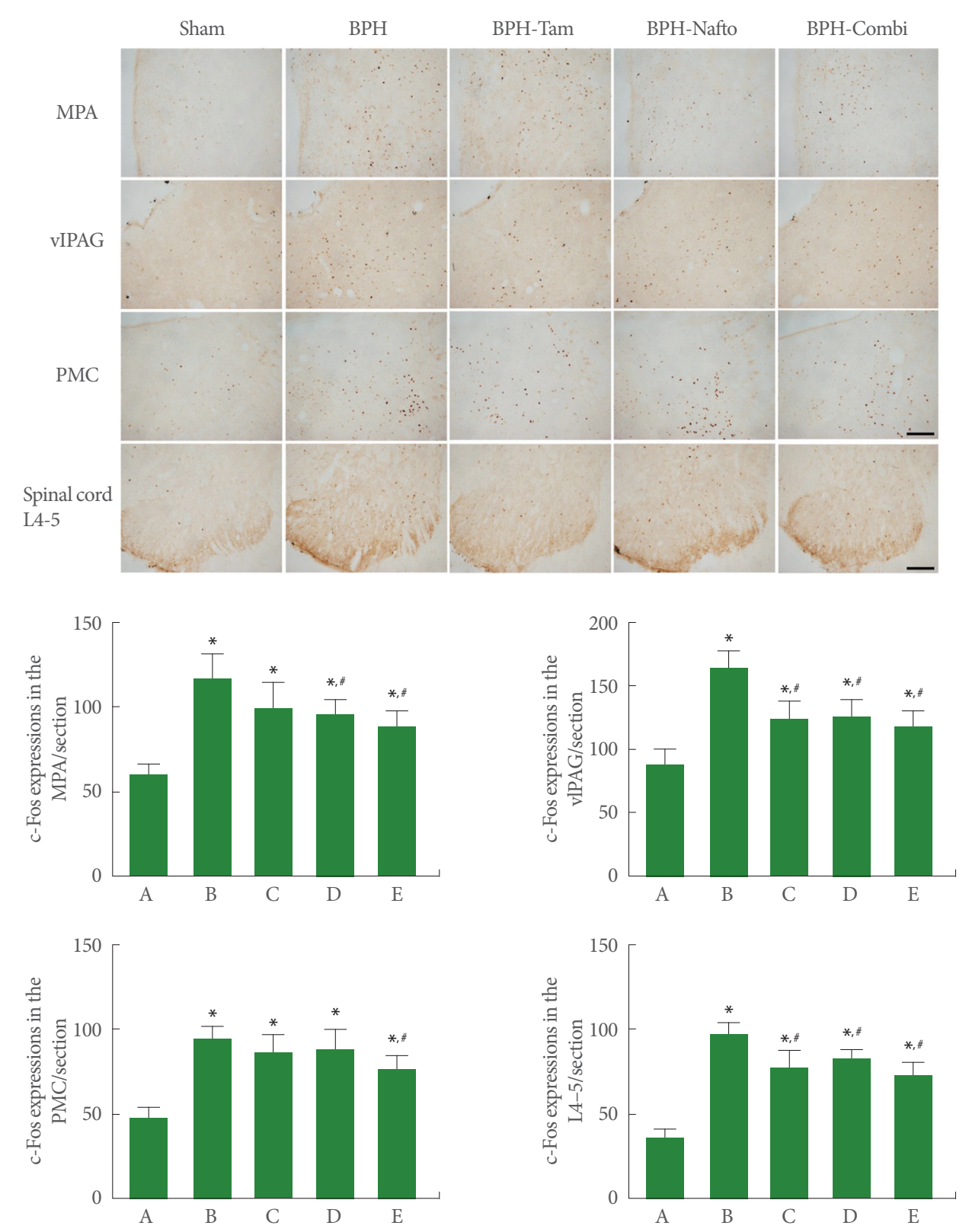

Fig. 2. c-Fos expressions in the neuronal voiding centers. Upper panel: photomicrographs of c-Fos-stained cells in neuronal voiding centers. The scale bar represents $150 \mu \mathrm{m}$ (MPA, medial preoptic area; vlPAG, ventrolateral periaqueductal gray; PMC, pontine micturition center) and $200 \mu \mathrm{m}$ (Spinal cord L4-5). Sham, sham-operation group; BPH, benign prostatic hyperplasia; BPH-Tam, BPH-induced and tamsulosin-treated group; BPH-Nafto, BPH-induced and naftopidil-treated group; BPH-Combi, BPH-induced and combination-treated group. Lower panel: number of c-Fos-stained cells in each group. A, sham-operation group; B, benign prostatic hyperplasia (BPH)-induced group; C, BPH-induced and tamsulosin-treated group; D, BPH-induced and naftopidil-treated group; E, $\mathrm{BPH}$-induced and combination-treated group. ${ }^{*} \mathrm{P}<0.05$ compared to the sham-operation group. ${ }^{*} \mathrm{P}<0.05$ compared to the $\mathrm{BPH}$-induced group.

increased by the induction of $\mathrm{BPH}(\mathrm{P}<0.05)$. Monotherapy with tamsulosin or naftopidil showed an inhibitory effect on NGF expression depending on the site in the neuronal voiding cen- ters of BPH rats $(\mathrm{P}<0.05)$. Combination therapy (tamsulosin with naftopidil) showed an even greater reduction in NGF expression at all sites of the neuronal voiding centers of $\mathrm{BPH}$ rats. 

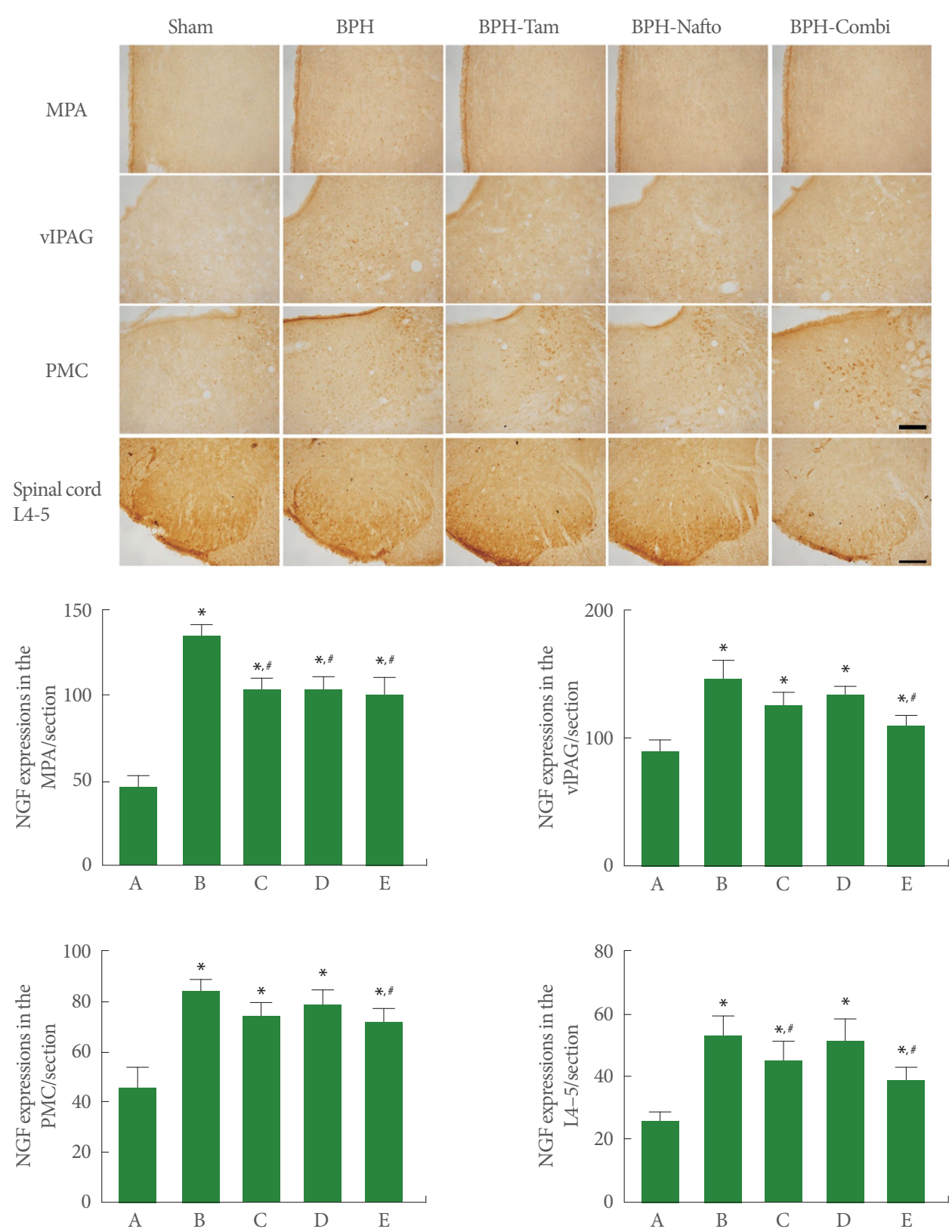

Fig. 3. Nerve growth factor (NGF) expressions in the neuronal voiding centers. Upper panel: photomicrographs of NGF-stained cells in neuronal voiding centers. The scale bar represents $150 \mu \mathrm{m}$ (MPA, medial preoptic area; vlPAG, ventrolateral periaqueductal gray; PMC, pontine micturition center) and $200 \mu \mathrm{m}$ (spinal cord L4-5). Sham, sham-operation group; BPH, benign prostatic hyperplasia; $\mathrm{BPH}$-Tam, BPH-induced and tamsulosin-treated group; $\mathrm{BPH}-\mathrm{Nafto}$, BPH-induced and naftopidil-treated group; $\mathrm{BPH}-\mathrm{Combi}$, $\mathrm{BPH}-$ induced and combination-treated group. Lower panel: number of NGF-stained cells in each group. A, sham-operation group; B, benign prostatic hyperplasia (BPH)-induced group; C, BPH-induced and tamsulosin-treated group; D, BPH-induced and naftopidiltreated group; $\mathrm{E}, \mathrm{BPH}$-induced and combination-treated group. ${ }^{*} \mathrm{P}<0.05$ compared to the sham-operation group. ${ }^{*} \mathrm{P}<0.05$ compared to the $\mathrm{BPH}$-induced group.

\section{NOS Expression in Neuronal Voiding Centers}

Photomicrographs of NADPH-d expression in the neuronal voiding centers are presented in Fig. 4. NOS expression in neu- ronal voiding centers was significantly increased by the induction of $\mathrm{BPH}(\mathrm{P}<0.05)$. Monotherapy with tamsulosin or naftopidil showed an inhibitory effect on NOS expression depend- 

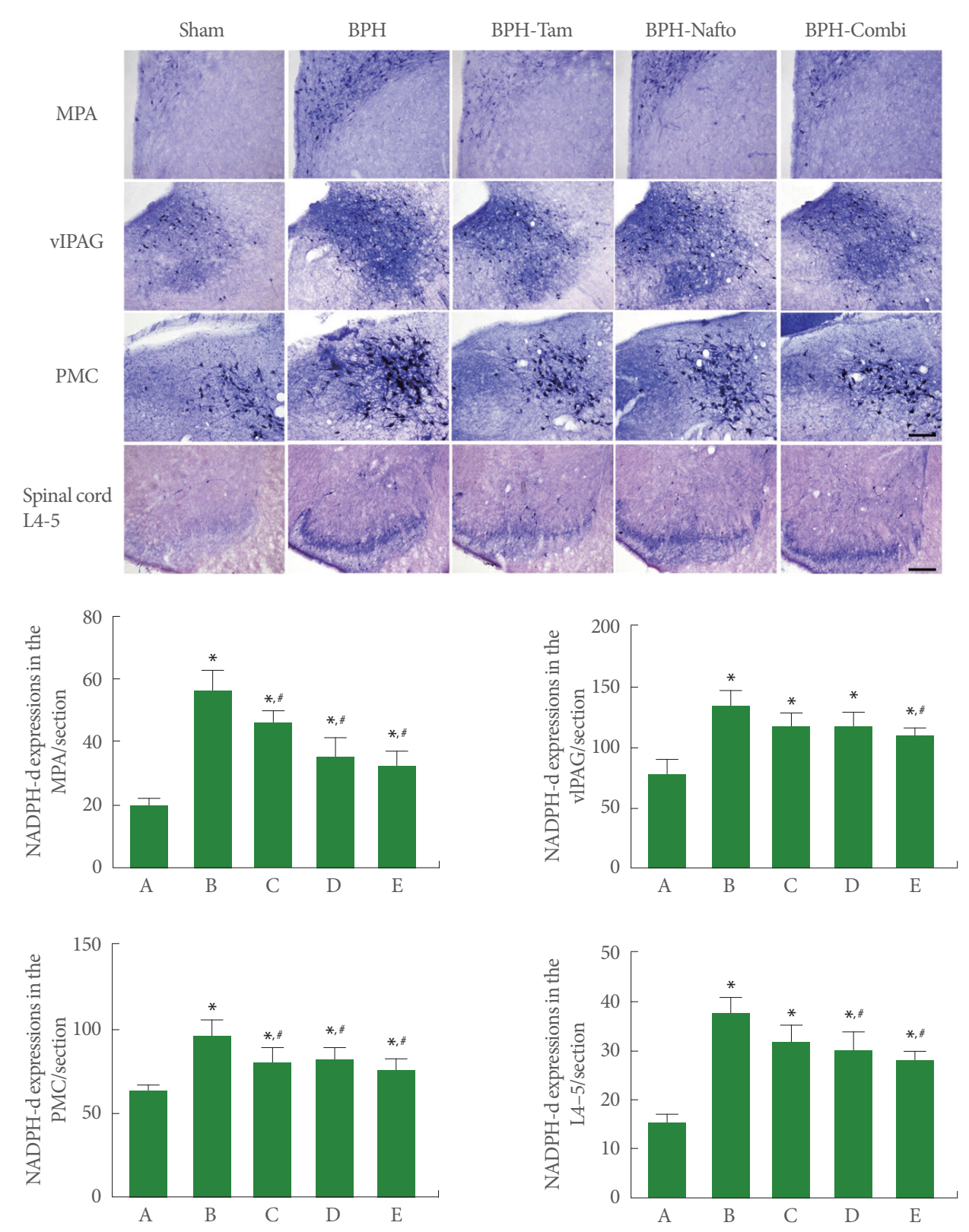

Fig. 4. Nitric oxide synthase (NOS) expressions in the neuronal voiding centers. Upper panel: photomicrographs of nicotinamide adenine dinucleotide phosphate-diaphorase (NADPH-d)-stained cells in neuronal voiding centers. The scale bar represents $150 \mu \mathrm{m}$ (MPA, medial preoptic area; vlPAG, ventrolateral periaqueductal gray; PMC, pontine micturition center) and $200 \mu \mathrm{m}$ (spinal cord L4-5). Sham, sham-operation group; BPH, benign prostatic hyperplasia; BPH-Tam, BPH-induced and tamsulosin-treated group; BPH-Nafto, BPH-induced and naftopidil-treated group; BPH-Combi, BPH-induced and combination-treated group. Lower panel: number of NADPH-d-stained cells in each group. A, sham-operation group; B, benign prostatic hyperplasia (BPH)-induced group; C, BPH-induced and tamsulosin-treated group; D, BPH-induced and naftopidil-treated group; E, BPH-induced and combinationtreated group. ${ }^{\star} \mathrm{P}<0.05$ compared to the sham-operation group. ${ }^{*} \mathrm{P}<0.05$ compared to the $\mathrm{BPH}$-induced group.

ing on the site in the neuronal voiding centers of $\mathrm{BPH}$ rats $(\mathrm{P}<0.05)$. Combination therapy (tamsulosin with naftopidil) showed an even greater reduction in NOS expression at all sites of the neuronal voiding centers of $\mathrm{BPH}$ rats. 


\section{DISCUSSION}

Exogenous testosterone supplementation has been repeatedly shown to induce prostate enlargement, and therapeutic drugs for BPH target prostate size [13]. Decreased voiding pressure and contraction time with enlargement of the prostate occurred following the administration of testosterone $[13,14]$. In the present study, voiding contraction pressure and interval contraction time were decreased by testosterone injections, demonstrating that $\mathrm{BPH}$ was induced by repeated testosterone injections.

For the treatment of bladder outlet obstruction by BPH, a1antagonists are the most widely used pharmacological agents [15]. Tamsulosin exhibits a dominant affinity to the alA-AR subtype compared to the $\alpha_{1 D}$-AR subtype, and tamsulosin exerts distinct actions on the prostate, external sphincter, and urethra [16]. Naftopidil has a 3 fold higher affinity for the $\alpha_{1 D^{-}}$ ARsubtype than for the alA-AR subtype, and naftopidil shows different effects on lower urinary tract symptoms because many a1D-ARs exist in the bladder neck [17]. Both a1-AR subtype antagonists alleviate bladder outlet obstruction symptoms, such as residual urine and urinary urge sensation, by reducing the prostate size [18]. Overactive bladder symptoms, including frequency, urgency, nocturia, and urgency incontinence, were decreased by a1-AR antagonists in clinical and animal studies $[12,19]$. Furthermore, al-AR antagonists increase bladder capacity and decrease frequency $[10,20]$. Each $\alpha 1-A R$ antagonist has unique properties based on its affinity profile and different effects on the central nervous system [17,21]. In the present study, combination therapy of tamsulosin with naftopidil enhanced voiding contraction pressure and interval contraction time. In contrast, monotherapy with tamsulosin or naftopidil did not exert a significant effect on voiding contraction pressure or the interval contraction time in $\mathrm{BPH}$ rats. These effects might be ascribed to the agonistic effects of tamsulosin and naftopidil in areas of the lower urinary tract such as the urethra, bladder, and prostate.

Voiding function is controlled by central micturition centers. The PMC plays an important role in controlling urinary bladder or urethral functions. During micturition, sympathetic motor neurons are suppressed and parasympathetic motor neurons are activated. PMC neurons directly activate parasympathetic preganglionic motor neurons, causing bladder contraction and sustained relaxation of the urethral sphincter [22]. vlPAG is important for controlling micturition through both af- ferent and efferent pathways. The efferent inhibitory signal passes through the PAG to the pons, and this excessive inhibitory signal triggers a reflex in the $\mathrm{PMC}$, resulting in urethral sphincter relaxation [23]. The PAG-PMC projection is thought to be involved in the micturition reflex. The MPA sends projections to the PMC that synapse on neurons directly though projections to the spinal cord [24]. In this context, voiding is under the control of brain areas such as the prefrontal cortex, anterior circulate cortex, insula, hypothalamus, and spinal cord [23].

Enhanced c-Fos expression represents neuronal activation [25], and noxious stimulation of the muscle, joint, and viscera increased the number of c-Fos-positive neurons in the vlPAG [26]. Chemical irritation of the bladder also enhanced c-Fos expression in the PAG and MPA of rats [27]. Nitric oxide (NO) regulates vascular smooth muscle tone and neurotransmission in the peripheral and central nervous systems [28]. The NO pathway is implicated in pathological bladder activity $[10,29]$. NO levels in the neuronal voiding centers also control lower urinary tract status [30]. NOS expression in neuronal voiding centers increased after cyclophosphamide injection, indicating that the induction of overactive bladder increased NO levels in the neuronal voiding centers [10]. In the present study, monotherapy with tamsulosin or naftopidil showed inhibitory effects on testosterone-induced c-Fos, NGF, and NOS expression depending on the site in the neuronal voiding centers. In particular, combination therapy of tamsulosin with naftopidil led to a greater reduction of c-Fos, NGF, and NOS expression at all sites in the neuronal voiding centers. These results suggest that cFos, NGF, and NOS in the neuronal voiding centers are involved in the modulation of micturition and that combination therapy more potently inhibits neuronal activation in the neuronal voiding centers of $\mathrm{BPH}$ rats.

In conclusion, combination therapy of tamsulosin and naftopidil may inhibit neuronal activity in the neuronal voiding centers to a greater extent than monotherapy with tamsulosin or naftopidil, and the enhanced inhibitory signal may relax the urethral and prostate smooth muscle. As combination therapy showed greater efficacy for the treatment of $\mathrm{BPH}$ than monotherapy, combination therapy can be considered as a novel therapeutic method for $\mathrm{BPH}$.

\section{ACKNOWLEDGEMENTS}

An earlier version of this study was presented at the SUFU (Society of Urodynamics, Female Pelvic Medicine and Urogenital 
Reconstruction) 2018 Winter Meeting, Austin, TX, USA, February 27-March 3, 2018.

\section{AUTHOR CONTRIBUTION STATEMENT}

- Full access to all the data in the study and takes responsibility for the integrity of the data and the accuracy of the data analysis: IG Ko, ST Cho

- Study concept and design: ST Cho

- Acquisition of data: L Hwang, JJ Jin, SH Kim

- Analysis and interpretation of data: L Hwang, KJ Chung, SH Kim

- Drafting of the manuscript: IG Ko

- Critical revision of the manuscript for important intellectual content: JW Jeon, JH Han

- Statistical analysis: JH Han, JJ Jin

- Obtained funding: ST Cho

- Administrative, technical, or material support: KJ Chung

- Study supervision: ST Cho, JW Jeon

\section{REFERENCES}

1. Ishii $K$, Sugimura Y. Identification of a new pharmacological activity of the phenylpiperazine d erivative naftopidil: tubulin-binding drug. J Chem Biol 2014;8:5-9.

2. Fowler CJ. Integrated control of lower urinary tract--clinical perspective. Br J Pharmacol 2006;147 Suppl 2:S14-24.

3. Ku JH, Ko DW, Cho JY, Oh SJ. Correlation between prostatic urethral angle and bladder outlet obstruction index in patients with lower urinary tract symptoms. Urology 2010;75:1467-71.

4. Kang M, Kim M, Choo MS, Bae J, Ku JH, Yoo C, et al. Association of high bladder neck elevation with urodynamic bladder outlet obstruction in patients with lower urinary tract symptoms and benign prostatic hyperplasia. Urology 2014;84:1461-6.

5. Kawai Y, Oka M, Yoshinaga R, Fuchikami C, Oyama T. Effects of the phosphodiesterase 5 inhibitor Tadalafil on bladder function in a rat model of partial bladder outlet obstruction. Neurourol Urodyn 2016;35:444-9.

6. Yuan J, Liu Y, Yang Z, Qin X, Yang K, Mao C. The efficacy and safety of alpha-1 blockers for benign prostatic hyperplasia: an overview of 15 systematic reviews. Curr Med Res Opin 2013;29:279-87.

7. Homma Y, Gotoh M, Yokoyama O, Masumori N, Kawauchi A, Yamanishi T, et al. Outline of JUA clinical guidelines for benign prostatic hyperplasia. Int J Urol 2011;18:741-56.

8. Kim HJ, Sun HY, Choi H, Park JY, Bae JH, Doo SW, et al. Efficacy and safety of initial combination treatment of an alpha blocker with an anticholinergic medication in benign prostatic hyperplasia patients with lower urinary tract symptoms: updated meta-analysis. PLoS One 2017;12:e0169248.

9. Kim SK, Seok H, Park HJ, Jeon HS, Kang SW, Lee BC, et al. Inhibitory effect of curcumin on testosterone induced benign prostatic hyperplasia rat model. BMC Complement Altern Med 2015;15: 380.

10. Kim SE, Shin MS, Kim CJ, Park JH, Chung KJ, Jung H, et al. Effects of tamsulosin on urinary bladder function and neuronal activity in the voiding centers of rats with cyclophosphamide-induced overactive bladder. Int Neurourol J 2012;16:13-22.

11. Jin LH, Park CS, Kim D, Choi BH, Park SH, Yoon SM, et al. Flow starting point and voiding mechanisms measured by simultaneous registrations of intravesical, intra-abdominal, and intraurethral pressures in awake rats. Int Neurourol J 2014;18:68-76.

12. Ko IG, Moon BM, Kim SE, Jin JJ, Hwang L, Ji ES, et al. Effects of combination treatment of alpha 1-adrenergic receptor antagonists on voiding dysfunction: study on target organs in overactive bladder rats. Int Neurourol J 2016;20(Suppl 2):S150-8.

13. Oudot A, Oger S, Behr-Roussel D, Caisey S, Bernabé J, Alexandre L, et al. A new experimental rat model of erectile dysfunction and lower urinary tract symptoms associated with benign prostatic hyperplasia: the testosterone-supplemented spontaneously hypertensive rat. BJU Int 2012;110:1352-8.

14. Miao M, Wang T, Liu J, Li Y, Fu Z, Tian S. The effect of Leonuri Herba alkaloids on senile BPH (male and female hormone induced) model rats. Saudi J Biol Sci 2017;24:630-3.

15. Lepor H, Tang R, Meretyk S, Shapiro E. Alpha 1 adrenoceptor subtypes in the human prostate. J Urol 1993;149:640-2.

16. Nascimento-Viana JB, Carvalho AR, Nasciutti LE, AlcántaraHernández R, Chagas-Silva F, Souza PA, et al. New multi-target antagonists of a1A-, a1D-adrenoceptors and 5-HT1A receptors reduce human hyperplastic prostate cell growth and the increase of intraurethral pressure. J Pharmacol Exp Ther 2016;356:212-22.

17. Nishino Y, Masue T, Miwa K, Takahashi Y, Ishihara S, Deguchi T. Comparison of two alpha1-adrenoceptor antagonists, naftopidil and tamsulosin hydrochloride, in the treatment of lower urinary tract symptoms with benign prostatic hyperplasia: a randomized crossover study. BJU Int 2006;97:747-51.

18. Griwan MS, Karthikeyan YR, Kumar M, Singh BJ, Singh SK. Comparative evaluation of naftopidil and tamsulosin in the treatment of patients with lower urinary tract symptoms with benign prostatic hyperplasia. Urol Ann 2014;6:181-6.

19. Athanasopoulos A, Gyftopoulos K, Giannitsas K, Fisfis J, Perimenis P, Barbalias G. Combination treatment with an alpha-blocker plus 
an anticholinergic for bladder outlet obstruction: a prospective, randomized, controlled study. J Urol 2003;169:2253-6.

20. Jeong MS, Lee JG. The role of spinal and peripheral alpha1- and alpha2-adrenoceptors on bladder activity induced by bladder distension in anaesthetized rat. BJU Int 2000;85:925-31.

21. Kim SE, Han JH, Ko IG, Kim CJ, Kim KH. Alpha1-adrenergic receptor antagonist tamsulosin ameliorates aging-induced memory impairment by enhancing neurogenesis and suppressing apoptosis in the hippocampus of old-aged rats. Animal Cells Syst. 2017;21: 404-11.

22. Vignes JR, De Seze M, Dobremez E, Joseph PA, Guérin J. Sacral neuromodulation in lower urinary tract dysfunction. Adv Tech Stand Neurosurg 2005;30:177-224.

23. Kitta T, Mitsui T, Kanno Y, Chiba H, Moriya K, Shinohara N. Brain-bladder control network: the unsolved 21st century urological mystery. Int J Urol 2015;22:342-8.

24. Rickey LM, Sarkey S, DonCarlos LL. Estrogen-sensitive projections from the medial preoptic area to the dorsal pontine tegmentum, including Barrington's nucleus, in the rat. Neurourol Urodyn 2008; 27:440-5.
25. Lee TH, Jang MH, Shin MC, Lim BV, Kim YP, Kim H, et al. Dependence of rat hippocampal c-Fos expression on intensity and duration of exercise. Life Sci 2003;72:1421-36.

26. Keay KA, Clement CI, Owler B, Depaulis A, Bandler R. Convergence of deep somatic and visceral nociceptive information onto a discrete ventrolateral midbrain periaqueductal gray region. Neuroscience 1994;61:727-32.

27. Chung IM, Kim YS, Sung YH, Kim SE, Ko IG, Shin MS, et al. Effects of acupuncture on abdominal leak point pressure and c-Fos expression in the brain of rats with stress urinary incontinence. Neurosci Lett 2008;439:18-23.

28. Ho MH, Bhatia NN, Khorram O. Physiologic role of nitric oxide and nitric oxide synthase in female lower urinary tract. Curr Opin Obstet Gynecol 2004;16:423-9.

29. Pandita RK, Persson K, Andersson KE. Capsaicin-induced bladder overactivity and nociceptive behaviour in conscious rats: involvement of spinal nitric oxide. J Auton Nerv Syst 1997;67:184-91.

30. Nazif O, Teichman JM, Gebhart GF. Neural upregulation in interstitial cystitis. Urology 2007;69(4 Suppl):24-33. 